REVUE DE L'INSTITUT FRANÇAIS D'HISTOIRE EN ALLEMAGNE

\section{Revue de I'IFHA}

Revue de l'Institut français d'histoire en Allemagne

$1 \mid 2009$

IFHA 1

\title{
La construction saint-simonienne du féminin entre 1829 et 1845 . Une histoire croisée des réseaux familiaux en rapport avec la Haskala berlinoise
}

\section{Paola Ferruta}

\section{CpenEdition}

\section{Journals}

Édition électronique

URL : http://journals.openedition.org/ifha/357

DOI : 10.4000/ifha.357

ISSN : 2198-8943

Éditeur

IFRA - Institut franco-allemand (sciences historiques et sociales)

Édition imprimée

Date de publication : 30 septembre 2009

Pagination : 181-186

ISSN : 2190-0078

Référence électronique

Paola Ferruta, « La construction saint-simonienne du féminin entre 1829 et 1845. Une histoire croisée des réseaux familiaux en rapport avec la Haskala berlinoise », Revue de l'IFHA [En ligne], 1 | 2009, mis en ligne le 07 février 2013, consulté le 20 avril 2019. URL : http://journals.openedition.org/ifha/357 ; DOI : 10.4000/ifha.357

Ce document a été généré automatiquement le 20 avril 2019.

(CIFHA 


\title{
La construction saint-simonienne du féminin entre 1829 et 1845 . Une histoire croisée des réseaux familiaux en rapport avec la Haskala berlinoise
}

\author{
Paola Ferruta
}

L'apport juif à la construction du féminin, ou plutôt celui des saint-simoniens concernés par la culture juive, a été au cœur de notre travail de recherche. La période considérée englobe la confrontation des saint-simoniens avec la «question féminine » dès son début jusqu'aux dernières années $d u$ mouvement. D'un point de vue méthodologique, l'approche historiographique et théorique adoptée est interdisciplinaire, relevant de l'histoire des femmes et du genre. La recherche porte aussi sur l'histoire socio-culturelle ainsi que, d'une part, sur l'anthropologie historique et religieuse, et d'autre part, sur l'histoire du discours. Ces choix ont aussi été motivés par le fait que, récemment, la recherche sur le saint-simonisme a gagné du point de vue méthodologique par l'apport de l'anthropologie historique. Le recours à la psycho-histoire, opérant ici avec des approches psychanalytiques et socio-psychologiques, nous a permis d'analyser les dynamiques du groupe saint-simonien. En outre, nous avons cerné le «matérialisme anthropologique ", comme défini par Walter Benjamin, à tendance politico-religieuse des intellectuels juifs saint-simoniens. Par ce concept, nous avons souligné d'un côté l'anthropologie des genres, et de l'autre l'intérêt pour la physiologie et l'anatomie structurant la vision saintsimonienne d'un individu social "homme-femme». L'anthropologie des genres et le symbole de l'androgyne/hermaphrodite saint-simoniens sont investis par le concept de "matérialisme anthropologique " d'un caractère matérialiste, ne le confinant pas seulement au domaine des idées mais en faisant un catalyseur de pratiques discursives de large envergure. 
Au niveau méthodologique, nous avons aussi eu recours à l'« histoire croisée » qui nous a permis d'identifier un réseau d'interrelations entre idées et personnes en France et en Allemagne dans la première moitié du XIXe siècle. Certains saint-simoniens juifs et leur famille en France étant économiquement et culturellement liés aux cercles berlinois marqués par le mouvement des Lumières et par la Haskala (mouvance juive des Lumières, 1770-1880), nous nous sommes penchées sur leurs échanges effectifs en envisageant également la question du genre. Conjointement, nous avons retracé les parcours biographiques et les expériences de certains saint-simoniens du début du XIXe siècle jusqu'en 1870. La recherche biographique autour des saint-simoniens et leur étude en tant que «cas » se croisent ici efficacement avec l'analyse du contexte où s'inscrivirent leurs parcours personnels et familiaux ainsi que l'examen de son évolution. À ce titre, nos sources principales sont les écrits privés (manuscrits, lettres et brouillons) et, dans une moindre mesure, les écrits publiés du groupe saint-simonien. La consultation de plusieurs archives à Paris, Berlin, Londres, Cracovie et New York et l'exhumation de sources originales manuscrites jusqu'alors ignorées ou inexplorées constituent une phase fondamentale de cette étude.

Après une brève introduction faisant le point entre autres sur l'état de la recherche à propos du saint-simonisme, le deuxième chapitre de notre travail est consacré à un aperçu historique des aspects particuliers de la chronologie du saint-simonisme. Notre excursus est conçu en tant qu'abrégé de l'histoire ramifiée du saint-simonisme en France et en Europe. Les juifs actifs au sein du saint-simonisme choisirent de suivre simultanément deux directions apparemment divergentes: d'un côté, celle d'une réflexion concernant le judaïsme, son rôle dans l'histoire religieuse de l'humanité et la mutation de l'auto-compréhension juive et d'un autre côté, celle d'une identification avec l'utopie socialiste dont le comte Claude-Henri de Saint-Simon avait légué les fondements idéologiques à un étroit cercle de fidèles au moment de sa mort en 1825. Bien que SaintSimon n'ait pas épargné de son regard critique l'orgueil du «sombre» peuple juif, mentionnant sa persécution et son inébranlable attente de l'«époque messiaque », il l'avait identifié comme le premier peuple prophétisant la vision saint-simonienne de l'âge d'or. Le Nouveau Christianisme, dernier ouvrage de Saint-Simon paru l'année de sa mort, jetait les bases d'une nouvelle religion qui aurait coïncidé avec une reconfiguration de la société, permettant aux capacités personnelles de tous ses membres de s'épanouir davantage ainsi que d'améliorer les conditions de la classe la plus pauvre. Pour SaintSimon comme pour ses élèves, la nécessité d'une reconstruction morale et matérielle de la société après la Révolution était perçue comme impérative. Aux prémices de la Révolution de Juillet, les saint-simoniens, opposants aux violences révolutionnaires et au républicanisme, avaient enrichi leur corpus doctrinal de réflexions sur la morale de la société et la condition de la femme à leur époque. Cela fut perçu dans un premier temps en tant qu'ensemble théorique et nouvelle religion, qui d'ailleurs, allait finir par inquiéter les autorités après l'instauration de la Monarchie de Juillet, dès que ces idées se propagèrent dans les quartiers ouvriers de Paris.

Le troisième chapitre de ce travail s'attarde sur le "culte de la Mère " des saintsimoniens. Nous y cernons l'apport de la tradition juive à la construction du sauveur féminin, amorcée par les adeptes du saint-simonisme. Ils évoquèrent une Femme-Messie, nommée plus tard "la Juive», qu'ils supposaient être l'incarnation du salut de l'humanité. Celle-ci deviendra rapidement le point pivot du débat conceptuel et des initiatives concrètes du mouvement. Ce culte, d'une part, s'inscrit dans le contexte de la 
dévotion mariale au XIXe siècle et d'autre part, est reconductible aux représentations préalables de la "Raison révolutionnaire " et de l'« Être suprême ». L'hypostase des représentations féminines au XVIIIe siècle, dont la matrice fut notamment athée ou hétérodoxe, est en France un phénomène antérieur au messie féminin vénéré un demisiècle plus tard par la secte saint-simonienne. En effet, le mouvement saint-simonien se définissait lui-même en tant qu' "Église»; d'un point de vue sociologique, il est cependant à qualifier de «secte ». D'où notre hypothèse : l'hypostase féminine, voire le sauveur juif féminin évoqué par les saint-simoniens, relève des diverses formes de vénération juive du féminin au sein des mouvements sectaires de protestation socioreligieuse dans l'Europe de l'Est au XVIIIe siècle et, comme le souligna Gershom Scholem en 1963, se distinguent nettement de la mariologie chrétienne. De même, la conception de la Femme-Messie propre aux membres juifs de la secte saint-simonienne s'appuyait sur une lecture ésotérique de la Genèse arborant un discours critique du statu quo religieux et socio-politique. La construction du féminin accomplie par les saintsimoniens juifs doit, de la sorte, être comprise comme "réponse juive ", telle qu'elle est définie par Charles Mopsik, à la mystique du sauveur féminin initiée par le courant enfantinien - c'est-à-dire la mouvance majoritaire au cœur du saint-simonisme, guidée à partir de 1831 par le chef charismatique Prosper Enfantin. Les différents rapports au féminin coexistant au sein de la secte saint-simonienne ainsi que les diverses images de la Mère ou, plus précisément, de la Femme-Messie, sont liés à la mémoire culturelle des membres respectifs. Ces rapports spécifiques à la Mère témoignent de leurs origines religieuses et de leur vécu individuel. En bref, l'idéal du moi du noyau du groupe eut une connotation féminine, particulièrement différenciée chez les adhérents juifs. Ces derniers refusaient le binarisme esprit-matière supposant une réification du féminin. Du reste, la Juive est une figure idéale née de l'activité phantasmatique groupale dans sa phase terminale où convergèrent deux éléments conceptuellement fondamentaux pour la secte saint-simonienne : messianisme et féminité.

Les deux derniers chapitres qui constituent la troisième partie de ce travail sont primordiaux afin d'exposer nos sources. Le « cas » du saint-simonien Gustave d'Eichthal, présenté dans le quatrième chapitre, nous apparait comme "idéal-typique " de cette tendance à donner au mouvement une empreinte juive. Celui-ci, comme d'autres saintsimoniens juifs, disposait d'un large réseau relationnel européen dont l'étude permet de poser un nouveau regard sur sa confrontation intellectuelle avec Prosper Enfantin, meneur du saint-simonisme. L'histoire croisée franco-allemande marquant le début du saint-simonisme met également en lumière l'activisme des femmes juives et de celles attachées culturellement au judaïsme (Mélanie, Félicie, Euphrasie Rodrigues et autres). Le vécu des saint-simoniens juifs montre de surcroît les différents processus d'intégration sociale et religieuse en France et en Allemagne. Questionnant ce qui a été défini comme symbiose judéo-allemande, nous approfondissons une relecture qui a déjà reçu des impulsions significatives au cours des dernières années, notamment grâce aux travaux menés par Julius H. Schoeps.

Dans le cinquième chapitre sont analysés les parallèles entre l'apologie du saintsimonisme par Olinde Rodrigues et le programme politico-religieux du mouvement diffusé en Allemagne par Eduard Gans, Heinrich Heine, Moritz Veit, Rahel et Karl August Varnhagen von Ense. Une vision religieuse particulière ; empreignait la pensée des représentants juifs de l'intelligentsia berlinoise de l'époque ; leur affinité avec le saintsimonisme fut marquée par une considération pour le christianisme primitif - idéalisé 
déjà à l'époque des Lumières mais particulièrement exalté ensuite par les romantiques différemment nuancée chez chacun de ces intellectuels. La vive admiration du berlinois Abraham Mendelssohn pour les «fondateurs (Urheber) de la religion chrétienne » est comparable à celle d'Eugène ou d'Olinde Rodrigues.

$\mathrm{Au}$ demeurant, durant le processus d'assouplissement des heurts sociaux dans la modernité, les saint-simoniens avaient désigné dans leur élaboration théorique la « femme » et le « juif» comme idéaux-types, aux antipodes politiques de l'ordre institué. La juxtaposition de l'homme juif tourné vers l'avenir avec la femme juive défavorisée, " non-émancipée » et nouveau messie de l'humanité, avait dans ce contexte, une fonction précise: l'intégration du saint-simonisme dans la modernité, tout en englobant la "différence " par rapport à cette même modernité. Dans cette mesure, le mouvement saint-simonien s'inscrivait aussi bien dans le processus historique que dans l'histoire universelle, comprise comme histoire du salut de l'humanité (la Heilsgeschichte thématisée par Gotthold Ephraim Lessing, bien connu des saint-simoniens). De plus, l'examen historique de la composante juive du saint-simonisme conduit à une nouvelle lecture de la sécularisation en France au XIXe siècle. Les saint-simoniens juifs, en tant qu'héritiers (probablement les plus éloignés) de la «révolution intellectuelle et séculaire » de la Haskala, fondaient leur "voie hédoniste " de l'acculturation sur des "idées, idéaux et fondements » (Shmuel Feiner, 2002), en bref, sur la nouvelle éthique politique et sociale préconisée par Claude-Henri de Saint-Simon. En dépit de leur assimilation à la société chrétienne environnante, ces saint-simoniens juifs contribuèrent significativement à la cristallisation d'une nouvelle auto-compréhension juive. Cette cristallisation prenait son essor dans le processus de sécularisation qui investissait alors la société et la pensée juives en France et en Allemagne. Il était question d'une « sécularisation mystique », telle qu'elle est définie par Michael Löwy, au sein de laquelle les composantes religieuses s'entremêlaient avec un discours économique et social radicalement réformiste.

Du point de vue de l'histoire de la culture et de l'histoire du discours, la religiosité saintsimonienne et le matérialisme anthropologique façonné surtout par les membres juifs de la secte autour de 1830, servaient de substrat sémantique à l'esquisse plus vaste de l'ordre socio-économique conçue par les saint-simoniens. Cette nouvelle ébauche de la société «co-variait» - au sens où l'entend N. Luhmann - avec le cheminement mouvementé mais, en fin de compte, ininterrompu de la France vers la Modernité. 